Original Research Paper

\title{
Iterative Feedforward Control for Bearing-Free Multibody Systems
}

\author{
Nicola Y. Bailey, Christopher Lusty and Patrick S. Keogh \\ Department of Mechanical Engineering, University of Bath, United Kingdom
}

Article history

Received: $19-08-2021$

Revised: 12-09-2021

Accepted: 15-09-2021

Corresponding Author:

Nicola Yvonne Bailey

Department of Mechanical

Engineering, University of

Bath, United Kingdom

E-mail: n.y.bailey@bath.ac.uk

\begin{abstract}
Automated machinery and robotics are commonly conventional multibody systems containing bearing components, which exhibit uncertain, discontinuous and complex tribological characteristics. These generate wear and fundamentally limit the precision of small scale motion due to the tribological effects being difficult to compensate for using model-based active control. However, they can be eliminated through the replacement of traditional bearing joints with flexure couplings, which offers a potential increase in the performance envelope. Initially a plain flexure coupling capable of large deformation is investigated, with a representative mathematical model derived based on large deformation Euler-Bernoulli theory which is validated using a bespoke experimental facility; proof of concept for the design of empirical controllers utilising experimental data is presented. Various designs of novel compound flexure couplings are conceived, comprising of multiple sections of spring steel. The presented compound flexure couplings are then characterised experimentally. A focused study of a two-compound flexure coupling-rigid body system is presented and the feasibility of generating open-loop feedfoward controllers from identified models is demonstrated in terms of accurate large displacement control. Including path correction in the presented control methodology reduces tracking errors by at least $62 \%$ and $71 \%$ in $(x, y)$ directions, respectively, for the cases considered.
\end{abstract}

Keywords: Feedforward Control, Flexure Coupling, Compound Flexure Coupling, Large Deformation

\section{Introduction}

Many automated systems used in robotic applications and manufacturing processes comprise of conventional mechanisms, made up of numerous rigid bodies connected through traditional translating and/or rotary joints. The efficiency, repeatability and precision of these automated systems are limited due to uncertain, discontinuous and complex tribological effects within the bearing joints, including friction, stiction, backlash, torque ripple and compliance. Degradation of the joint performance also occurs, typically over a period of operation due to wear and if a mechanism has multiple joints, the effects of the individual joints will be compounded, producing complex overall tribological effects. Therefore, the system's capability to accurately follow a prescribed trajectory, either a displacement, velocity or acceleration profile, is limited.

Currently, automated systems are generally used in industrial processes where the uncertainty in the small scale motion is inconsequential, such as the assembly and inspection of manufactured products. However, for high added-value components of complex systems, including those in the automotive and aerospace industries, it is imperative that manufacturing techniques are accurately controlled with repeatable motion. Therefore, introducing automation through robotics and sensing for these high added-value components must not compromise the current achievable precision and repeatability. However, it is difficult to compensate for the complex and uncertain nonlinear tribological effects in traditional bearing joints with active control.

A novel approach to eliminate the source of small scale uncertain and discontinuous effects, is through new designs of multibody mechanisms, with joints comprising of flexure couplings, or compact deformable structures. These permit movement between the rigid bodies in the mechanism through elastic deformation, thus providing precise and frictionless motion in their workspace (Smith, 2000; Howell, 2001), resulting in a smooth trajectory path. Additional benefits are that flexure couplings do not require lubrication nor are they subject to wear. Furthermore, the overall mechanisms are less complicated with a reduced number of parts. However, systems containing flexure couplings do introduce additional 
degrees of freedom in which the system can experience dynamic motion. Although nonlinear, the mechanical characteristics are determinate. Hence, efficient, precise and repeatable motion of a mechanism containing flexure couplings should be feasible when regulated under suitable control strategies and actuation. These systems could be useful in robotic applications and manufacturing.

In micro- and nano-positioning mechanisms, flexure couplings undergoing very small deflections are commonly used, for example see Chen et al. (2020) and their ability to achieve robust high precision movement without suffering from parasitic motion (Herfst et al., 2015; Fantner et al., 2006) is key to their performance. Flexure couplings capable of large deflections have been implemented in robotic hands for grasping and dexterous manipulation (Liarokapis and Dollar, 2016; Odhner et al., 2014) as well as objective identification (Spiers et al., 2016).

Predictions for systems comprising flexure joints and rigid bodies in series, forming pseudo-kinematic chains, can be obtained through simplified mathematical models. These models are typically based on pseudo-rigid-body models where the flexure couplings are represented as a serial-chain of rigid bodies, with passive elastic joint connections (Howell and Midha, 1994). Alternatively, an equivalent pin model has been studied which accounts for the non-stationary centre of rotation and varying radius in the flexure coupling (Guo and Lee, 2013). However, the flexure coupling has to be limited in length to obtain realistic predictions Venkiteswaran and Support (2016). The case of a four-bar link pendulum excited by gravity alone was examined by Lobontiu (2001), comprising two rigid links and two flexure couplings, however the flexure coupling deformation was assumed to be small. The effect of the flexure coupling geometry on its performance was examined by Ma et al. (2020) on typical small deformation notched flexure hinges, concluding the design and optimisation of notch shapes can have a significant effect on behaviour. Topology optimisation of flexure hinges with regards to stress constraints whilst maintaining the desired motion found that the results differ from the conventional hinges topologies (Liu et al., 2017).

To achieve accurate predictions for a mechanism incorporating large deformation flexure couplings, the higher order nonlinear elastodynamics of the flexure couplings must be included. Steady state models based on nonlinear cantilever beams experiencing an external force, either tip concentrated and/or distributed loads, have been studied extensively, for example see Wang et al. (2008); Kimiaeifar et al. (2011); Argyris and Symeonidis (1981); Odhner and Dollar (2012); Rao et al. (1986); Shvartsman (2013); Ma and Chen (2016). However, there are limited studies of these being connected to rigid bodies, forming pseudo-kinematic chains. Recently, leading research presented a non-linear analytical approach, based on theories of bending (Henning et al., 2021) under large deflections together with shear and lateral contraction in a unified form (Henning and Zentner, 2016), to compute the elasto-kinematic properties of planar compliant mechanisms. Accurate and fast analysis of multiple planar compliant mechanisms with distributed or concentrated compliance is achieved. A plain large deformation flexure coupling consisting of a single section of spring steel has been investigated by Bailey et al. (2018) for precision control in a two-flexure coupling-rigid body system. The control methodology was derived from a bespoke large deformation nonlinear mathematical model and results showed the advantages of flexure couplings included elimination of high frequency behaviour associated with the excitation of the nonlinear effects in conventional bearing components. Thus demonstrating flexure couplings have the potential to replace traditional revolute joints in robotic and other automated systems, provided they can sustain dynamic loading envelopes.

This study considers the large deformation of compound Flexure Couplings (CFs), comprising of multiple sections and complex geometries, together with open-loop control methodologies for high precision multibody systems, based on identified models from experimental data. This control approach negates the need for complex and expensive models to be formulated for CFs, while still providing trajectory control of equal accuracy. Initially, a Plain Flexure coupling $(\mathrm{PF})$ is examined, with a nonlinear mathematical model to demonstrate the challenges of modelling CFs and provides a proof of concept for designing open-loop feedforward controllers from experimental data. The openloop approach is preferred over traditional closed loop PID control due to potential problems associated with nonlinear behaviour. The CF designs are presented and their characterisation is assessed through experimental testing. A double-CF-rigid body system incorporating these flexure couplings is introduced with control methodology developed, based on an open-loop feedforward controller derived from experimental data. This methodology accounts for the nonlinear elastodynamics introduced by CFs. As such the designed controllers have the potential to regulate the actuation and achieve precise motion tracking, particularity when path correction is incorporated. The accuracy of controlling the trajectory of a multi-rigid body system containing CFs and PFs is examined through the use of a bespoke experimental facility to demonstrate their potential in motion control systems. In principle, the fatigue life of flexures could be assessed for number of cycles against deflection amplitude. Flexures used in voice coil actuators are rated for fatigue in this manner. However, the flexure design and system feedforward control are the main focus of this study.

\section{Plain Flexure Coupling}

A PF which consists of monolithic material with suitable elastic properties for large elastic deformation is investigated. A mathematical model for the PF is derived using nonlinear Euler-Bernoulli large deformation beam theory (Bailey et al., 
2018). The PF model details are introduced in this section to demonstrate the difficulty associated with modelling more complex (e.g., cross-) flexure coupling designs.

A schematic of a PF is given in Fig. 1a, with the corresponding experimental PF shown in Fig. 1b, where the left hand side is fixed whilst the right hand side is actuated. The model is formulated assuming that a pair of forces and moment $\boldsymbol{F}_{f}=\left(F_{f x}, F_{f y}, F_{f f}\right)^{T}$ are applied to the tip of the flexure at the centreline; $F_{f x}$ and $F_{f y}$ are imposed in the horizontal and vertical directions, respectively, regardless of the PF deflection. The PF tip deflection is denoted by the vector $\boldsymbol{x}_{f}=\left(x_{f}, y_{f}, \theta_{f}\right)^{T}$ and the arc length coordinate $s=\left[0, l_{f}\right]$ is introduced in two-dimensional Euclidean space along the flexure centreline, with Cartesian coordinates at a given $s$ defined by:

$$
x(s)=\int_{0}^{s} \cos (\theta(u)) d u, \quad y(s)=\int_{0}^{s} \sin (\theta(u)) d u .
$$

Large deformation Euler-Bernoulli theory is utilised, in which it is assumed that deformation occurs primarily through bending with the axial and shear deformations assumed negligible due to the PF having significantly larger width and length than thickness. The bending moment $M(s)$ at given length $s$ is assumed to be proportional to the corresponding curvature, leading to the governing equation:

$$
\begin{aligned}
E I \frac{d \theta}{d s} & =M(s) \\
& =-F_{f x}\left(y_{f}-y(s)\right)+F_{f y}\left(x_{f}-x(s)\right)+F_{f \theta},
\end{aligned}
$$

where, $E$ is the Young's modulus and $I$ the second moment of area.

By differentiating Eq. (2) with respect to the arc length $s$ and using relations $d x / d s=\cos \theta$ and $d y / d s=\sin \theta$ from (1), the governing equations for the PF are found. Setting $\theta=z_{1}$ and $d \theta / d s=z_{2}$, results in a set of first order ordinary differential equations:

$$
\begin{aligned}
& \frac{d z_{1}}{d s}=z_{2}, \quad \frac{d z_{2}}{d s}=\frac{1}{E I}\left(F_{f x} \sin z_{1}-F_{f y} \cos z_{1}\right), \\
& \frac{d x}{d s}=\cos z_{1}, \frac{d y}{d s}=\sin z_{1} .
\end{aligned}
$$

Corresponding boundary conditions are given by:

$$
z_{1}(0)=0, z_{2}\left(l_{f}\right)=\frac{F_{f \theta}}{E I}, x(0)=0, y(0)=0,
$$

since it is assumed the PF is fixed at $s=0$ with zero angle and the curvature at the free end is as given in Eq. (2). This system of equations demonstrates that the PF can be characterised by the angle alone.

The system of Eq. (3) and (4) can be solved with a suitable initial estimate, then using a finite difference method implementing a three-stage Lobatto IIIa formula. An interesting phenomenon of this problem is that there is a many-to-many relationship between the pair of forces and moment applied to the PF, $\boldsymbol{F}_{f}$ and the resulting tip deflection, $\boldsymbol{x}_{\boldsymbol{f}}$. Figure 2 shows multiple solutions for the set of forces and moments $\boldsymbol{F}_{f}=(10 \mathrm{~N},-1000 \mathrm{~N}, 10 \mathrm{Nm})^{T}$ when different initial estimates are used. Alternatively, multiple sets of forces and moments applied to the PF can result in the same tip position. This phenomenon leads to the difficulty of modelling multi-sectioned compound Flexure Couplings (CFs), such as those in Fig. 3 for parallel and cross-over sections. This is due to the distribution of the external force $\boldsymbol{F}_{f}$ at the tips of each of the sections, $\boldsymbol{F}_{f 1}$ and $\boldsymbol{F}_{f 2}$, being unknown. An additional complication is that the sections may not be placed in the same plane as each other, especially in the cross-over design.

To validate the PF mathematical model, a bespoke research facility shown in Fig. 4 was used. The PF was made of fully hardened grade 301 stainless steel, which has a Young's modulus of $E=193 \times 10^{9} \mathrm{Nm}^{-2}$ and has dimensions of thickness of $0.4 \mathrm{~mm}$ and length and width of $30 \mathrm{~mm}$, giving a second moment of inertia of $I=1.6 \times 10^{-13} \mathrm{~m}^{4}$. Clamping the PF at both ends between blocks of aluminium together with incorporating dowling rods, prevented any unwanted movement. Actuation was provided by a lead screw actuator, which remains under compression at all times, imposes the force $\boldsymbol{F}_{f}$ onto the PF tip, through a linkage mechanism. A linear bearing was used to support the actuator rod to minimise the effects of side loading. The linkage mechanism was modelled as a series of rigid bodies and perfect hinge joints as in Fig. 4 and assumed to be massless; $l_{0}$ is the length of the actuator extension from the linear bearing to the first deep groove ball bearing; $l_{1}$ is the length of the rigid body between the two deep groove ball bearings, which has angle $\phi$ to the horizontal; $l_{2}$ is the length between the deep groove ball bearing and vertical rigid body, which has angle $\theta_{f}$ to the horizontal; $l_{v}$ is the length between the laser tracker targets; and $l_{p}$ is the distance between the PF tip and the upper laser tracker target.

The effect of an actuator force of component $F_{0}$ is to impose an equivalent force $\boldsymbol{F}_{f}$ on the PF tip. By equilibrium:

$$
\begin{aligned}
F_{f x}= & F_{0}, \\
F_{f y}= & F_{0} \tan \phi, \\
F_{f \theta}=\frac{F_{0}\left(l_{2} \sin \left(\theta_{f}-\phi\right)+l_{v} \cos \phi\left(\theta_{f}-\phi\right)\right)}{\cos \phi} & \quad+F_{f y} l_{p} \cos \theta_{f}-F_{f x} l_{p} \sin \theta_{f} .
\end{aligned}
$$

The angle $\phi$ and actuator extension $l_{0}$ are defined by the geometry of the system:

$$
\begin{aligned}
& l_{0}-\kappa+l_{1} \cos \phi=x_{f}+\left(l_{p}-l_{2}\right) \cos \theta_{f}+l_{v} \sin \theta_{f}, \\
& l_{1} \sin \phi+l_{v} \cos \theta_{f}=y_{f}+\left(l_{p}-l_{2}\right) \sin \theta_{f}+l_{v},
\end{aligned}
$$

where $\kappa$ is the offset of the linear bearing to the fixed end of the PF, as shown in Fig. 4. 
a)

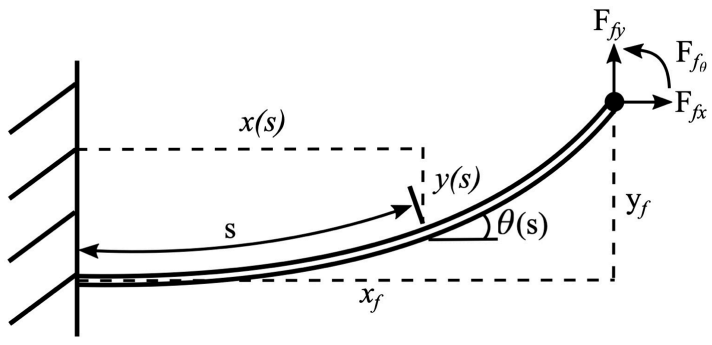

b)

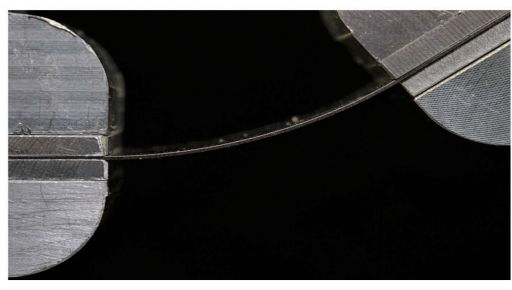

Fig. 1: (a) Schematic diagram for a PF with force $\boldsymbol{F}_{f}=\left(F_{f x}, F_{f y}, F_{f \theta}\right)^{T}$ imposed at the tip and (b) Image of a $\mathrm{PF}$ fixed on the left and actuated on the right

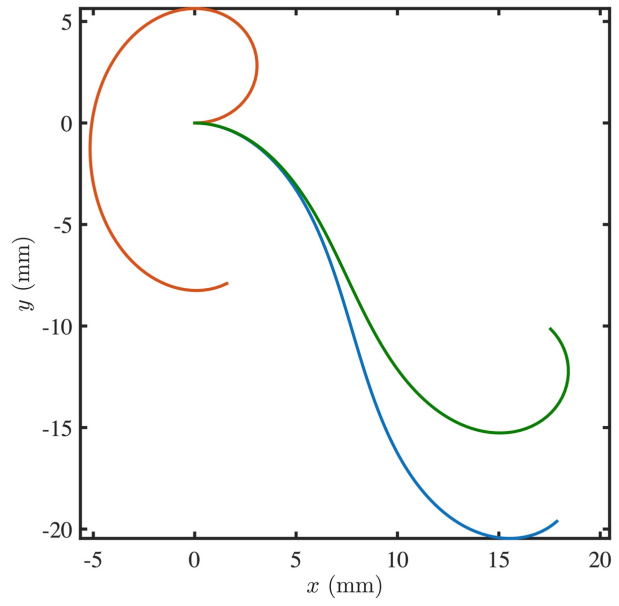

Fig. 2: Prediction of possible $\mathrm{PF}$ deflections with imposed $\boldsymbol{F}_{f}=(10 \mathrm{~N},-1000 \mathrm{~N}, 10 \mathrm{Nm})^{T}$ for different initial solution estimates

The input to the mathematical model is the actuator force component $F_{0}$, however, in the experimental facility the lead screw actuator does not have force sensing capabilities, though does have inbuilt potentiometers to identify the actuator extension $l_{0}$. Therefore, for control purposes $F_{0}$ is implicit with the input being the actuator extension $l_{0}$ and the output the deflection of the PF. Thus, experimental results were obtained by extending the actuator by increments of $5 \mathrm{~mm}$ and recording the static position of the system using a 3D laser tracker, which had an accuracy of $\pm 0.02 \mathrm{~mm}$, by utilising the four laser tracker targets shown in Fig. 4. a)

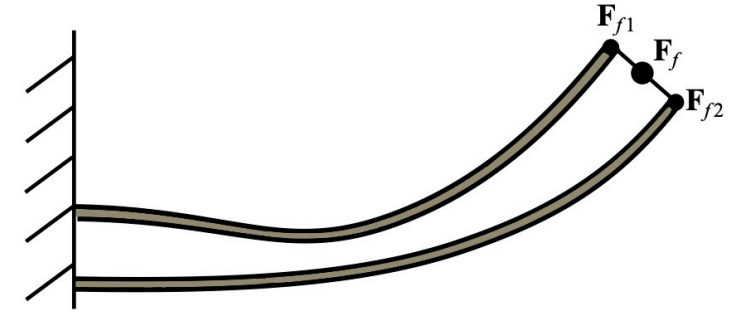

b)

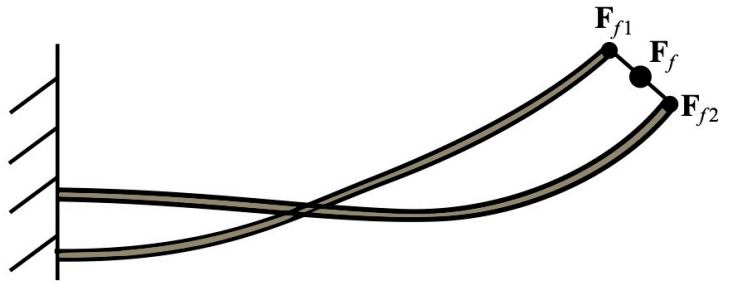

Fig. 3: Schematic side view for multi-sectioned CFs with (a) Parallel and (b) Cross-over design with external force $\boldsymbol{F}_{f}$ and forces on the tip of the sections $\boldsymbol{F}_{f 1}$ and $\boldsymbol{F}_{f 2}$

From the measured positions of the four targets, the PF tip deflection, $\left(x_{f}, y_{f}, \theta_{f}\right)^{T}$, can be identified. Corresponding theoretical predictions of the deformed PF were found by inputting a range of forces into the mathematical model and computing the PF tip deflection and actuator extension for the equivalent range of tip angle $\theta_{f}$. Results for the actuation configuration of the PF in Fig. 4, are shown in Fig. 5. These demonstrate that for a given actuator extension, $l_{0}$, there is a single PF tip deflection, $\left(x_{f}, y_{f}, \theta_{f}\right)^{T}$, showing good agreement between the theoretical predications and experimental results.

Therefore, the experimental results shown in Fig. 5 can be utilised in a look-up table to identify the actuator extension, $l_{0}$, needed to be imposed on the experimental facility to achieve one prescribed coordinate of the tip deflection position. For a system containing two PFs in series, where each PF is deformed by an independent actuator, two coordinates $\left(x_{p}, y_{p}\right)$ at the end of the system can be prescribed. The associated look-up tables, each consisting of the two actuator extensions and end coordinates of the system are computed using the PF tip deflections in Fig. 5 and geometry of the system. Therefore, an open-loop feedforward control methodology can be implemented which does not require a complex mathematical model to be used in its formulation.

\section{Compound Flexure Coupling}

The initial investigations of the PF, have shown that if the relationship between the actuator extension $l_{0}$ and the PF tip deflection are known and there is a one-toone relationship for the actuation mechanism, then the PF tip deflection can be controlled. It is asserted that similar methodology can be used for a CF of similar dimensions, where each section of the $\mathrm{CF}$ is represented by a PF. However, due to the difficulty in deriving realistic and accurate models for CFs, the 
relationship between the actuator extension $l_{0}$ and CF tip deflection are assessed through experimental testing in this study. The choice of CF design is not unique and many options are available with varying levels of complexity. Rather than analysing every design mathematically, the approach taken was to use system identification based on measured characteristics to generate models.

Three different $\mathrm{CF}$ designs were investigated which provide movement in two-dimensions and have the cross-over point in a central position, as shown in Fig. 6 . These were constructed from fully hardened grade 301 stainless steel sections; $\mathrm{CFa}$ has three equal width sections, $\mathrm{CFb}$ has a middle section having twice the width of the outer sections and $\mathrm{CFc}$ has two equal width sections. Each section has a length of $30 \mathrm{~mm}$ and a thickness of $0.4 \mathrm{~mm}$ and each design has an overall width of $30 \mathrm{~mm}$. Spacers of height $2 \mathrm{~mm}$ were used to create the vertical separation, with two positioned between the sections at both ends, giving a total separation of $4 \mathrm{~mm}$.

Modifications to the CF designs shown in Fig. 6 are investigated for the triple-section configurations $(\mathrm{CFa}$ and $\mathrm{CFb}$ ) when they have a common end point instead. The four possible modifications are shown in Fig. 7 for $\mathrm{CFa}$. Suffices $\mathrm{m} 1$ and $\mathrm{m} 3$ denote having the cross-over point at the fixed end with two and one section(s) on the upper side, respectively. Suffices $\mathrm{m} 2$ and $\mathrm{m} 4$ denote having the cross-over point at the actuated end with two and one section(s) on the upper side, respectively. Modifications for the double-section CF were not investigated as these arrangements lead to torsional effects and significant out of plane motion.

\section{Compound Flexure Characterisation}

Each of the $\mathrm{CF}$ designs were studied to identify the $\mathrm{CF}$ tip deflection as a function of actuator extension $l_{0}$. The actuator rod was retracted and extended in increments of $5 \mathrm{~mm}$ to $\pm 45 \mathrm{~mm}$ from the horizontal position, where $\theta_{f}=0$ at the reference extension of $0 \mathrm{~mm}$. At each increment the deflection of the CF was inferred from the 3D laser tracker target measurements.

Figure 8 shows all $\mathrm{CF}$ and $\mathrm{PF}$ tip positions for actuator extensions over a range of $\pm 45 \mathrm{~mm}$ together with the differences between the PF and CF tip positions, $\left(x_{P F}-x_{C F}, y_{P F}-y_{C F}\right)$. The results show that designs $\mathrm{CFa}, \mathrm{CFb}$ and $\mathrm{CFc}$ have a similar tip position, following the trend of the PF profile, but with a horizontal offset. The difference is between $0.2-0.6 \mathrm{~mm}$ across the full actuator stroke and arises due to the CFs having a shorter length along the centreline in the $x$ direction arising from the initial curvature of the sections due to the vertical separation at the clamped ends.

Figures $8 \mathrm{~b}$ and $\mathrm{c}$ indicate that the cross-over point of the CFs has a significant effect on the CF performance, with modifications $\mathrm{m} 1$ and $\mathrm{m} 3$ having similar behaviour, as do $\mathrm{m} 2$ and $\mathrm{m} 4$. When the cross-over point is at the fixed end for modifications $\mathrm{m} 1$ and $\mathrm{m} 3$, Fig. $8 \mathrm{~b} 1$ shows the flexure behaves in a similar way as an unmodified design, but with a greater range of motion for the same actuator extension range. This results in the difference between the $\mathrm{PF}$ and CF tip positions Fig. $8 \mathrm{~b} 2$ becoming larger, further from the horizontal position. Placing the cross-over point at the actuated end for modifications $\mathrm{m} 2$ and $\mathrm{m} 4$ (Fig. 8c1), leads to a smaller deflection of the $\mathrm{CF}$ for the same actuator extension range with significantly different behaviour. Figure $8 \mathrm{c} 2$ highlights the sharp and distinct changes at the horizontal position, most likely due to a buckling effect. Thus, for smooth controlled motion, CFs with no modification or modifications $\mathrm{m} 1 / \mathrm{m} 3$ would be ideal.

The characteristics of $\mathrm{CFa}$ and $\mathrm{CFb}$ and their respective modifications, are very similar, thus only $\mathrm{CFa}$ will be examined in subsequent investigations. Figures 9 and 10 give the flexure coupling deformations for the minimum and maximum actuator extension $( \pm 45 \mathrm{~mm})$ for $\mathrm{CFa}, \mathrm{CFc}$ and $\mathrm{PF}$ and $\mathrm{CFam} 3$ and CFam4, respectively. Figure 9 shows that the sections in $\mathrm{CFa}$ and $\mathrm{CFc}$ have similar deformations, with all sections having unidirectional bending. However, $\mathrm{CFc}$ experiences torsional effects due to the design imposing a moment on the flexure clamp at the actuated end. This causes out of plane motion, which will be amplified when additional CFs and rigid bodies are connected in series. It is for this reason that the design $\mathrm{CFc}$ was not taken further. To prevent torsional effects, a moment on the actuated flexure clamp must be avoided; designs must be symmetrical about the mid-point of the flexure coupling width. The effect of modifying the cross-over point to be at an end of the CF gives rise to sections having either unidirectional or multidirectional bending, depending on the section orientation as shown in Fig. 10. For example, for CFam4, when there is minimum actuator extension the sections that join the cross-over point from below has multidirectional bending, whereas the section that joins the cross-over point from above has unidirectional bending. The converse is true for the case of maximum actuator extension.

\section{Two Flexure Coupling Rigid Body System}

Having characterised the different CF designs, open-loop feedforward control methodologies can be formulated and implemented on the research facility to examine the resultant achievable precision for the $\mathrm{CF}$ designs. Figure 11 shows the experimental facility comprising of two CFs interconnected between two nominally rigid body links, consisting of lengths of aluminium profile and flexure clamps. The first $\mathrm{CF}$ is fixed to an aluminium profile support structure connected to a bedplate. Both CFs are deflected by lead screw actuators and linkage mechanisms, with linear bearings used to minimise side loads. The first actuator is fixed within the support structure while the second actuator is attached to the first rigid body link. 

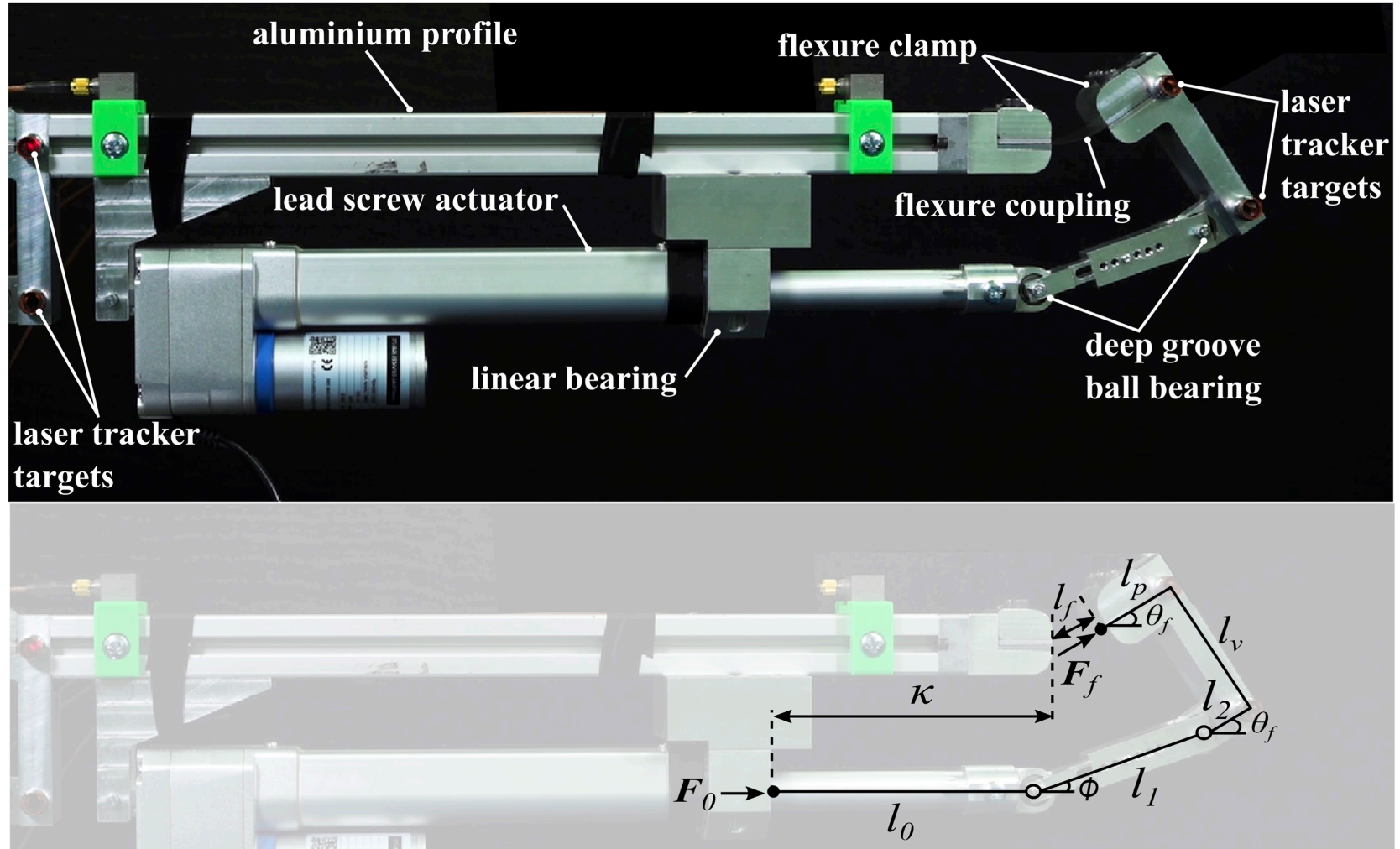

Fig. 4: Experimental facility for single flexure coupling with CAD diagram showing dimensions of the actuation mechanism
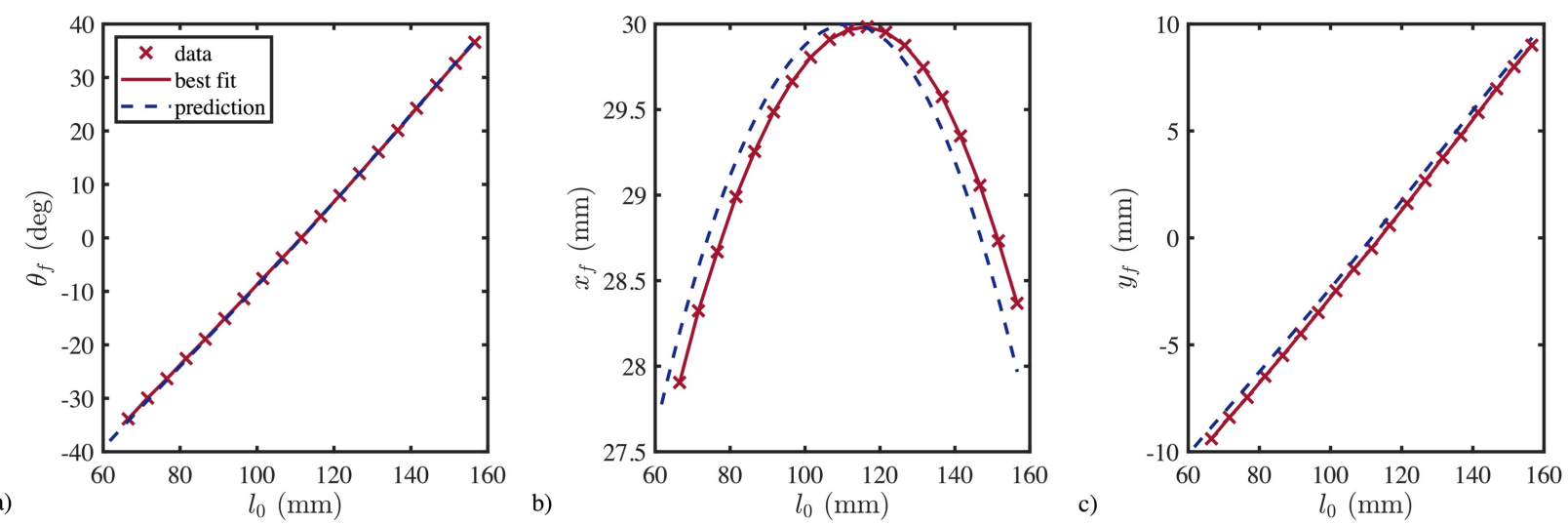

Fig. 5: Theoretical prediction (- -) together with experimental data $(\times)$ and best fit line (-) for a PF when the actuator is extended in increments of $5 \mathrm{~mm}$; (a) Tip angle $\theta_{f}$ against actuator extension $l_{0}$, (b) Horizontal tip coordinate $x_{f}$ against actuator extension $l_{0}$ and (c) Vertical tip coordinate $y_{f}$ against actuator extension $l_{0}$

\section{Control Methodology}

To precisely control the two-CF-rigid body system, bespoke controllers are developed for each CF design. Open-loop feedforward control methodologies are formulated from the experimental data such that the end position of the two-CF-rigid body system follows a prescribed trajectory. It is assumed that both $\mathrm{CF}$ joints behave identically, given that they have the same design. 


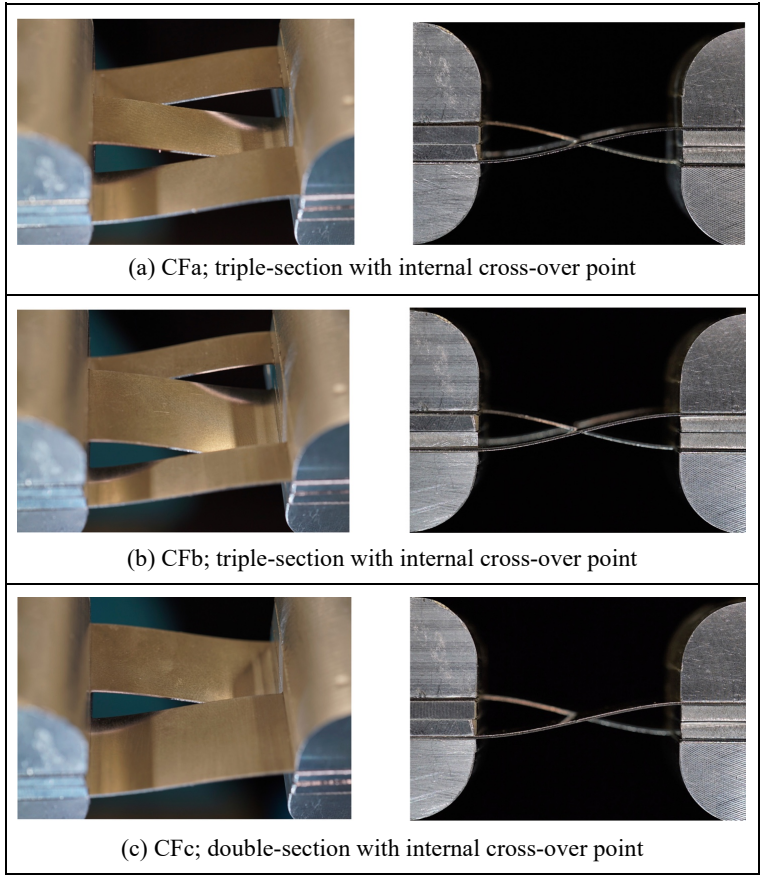

Fig. 6: Designs of CF having internal cross-over points

Therefore, the end position of the two-CF-rigid body system can be calculated, using the geometry of the system, over the range of both actuator extensions $-45 \mathrm{~mm} \leq\left(l_{01}, l_{02}\right) \leq 45 \mathrm{~mm}$, where subscripts 1 and 2 denote the first and second actuator, respectively.
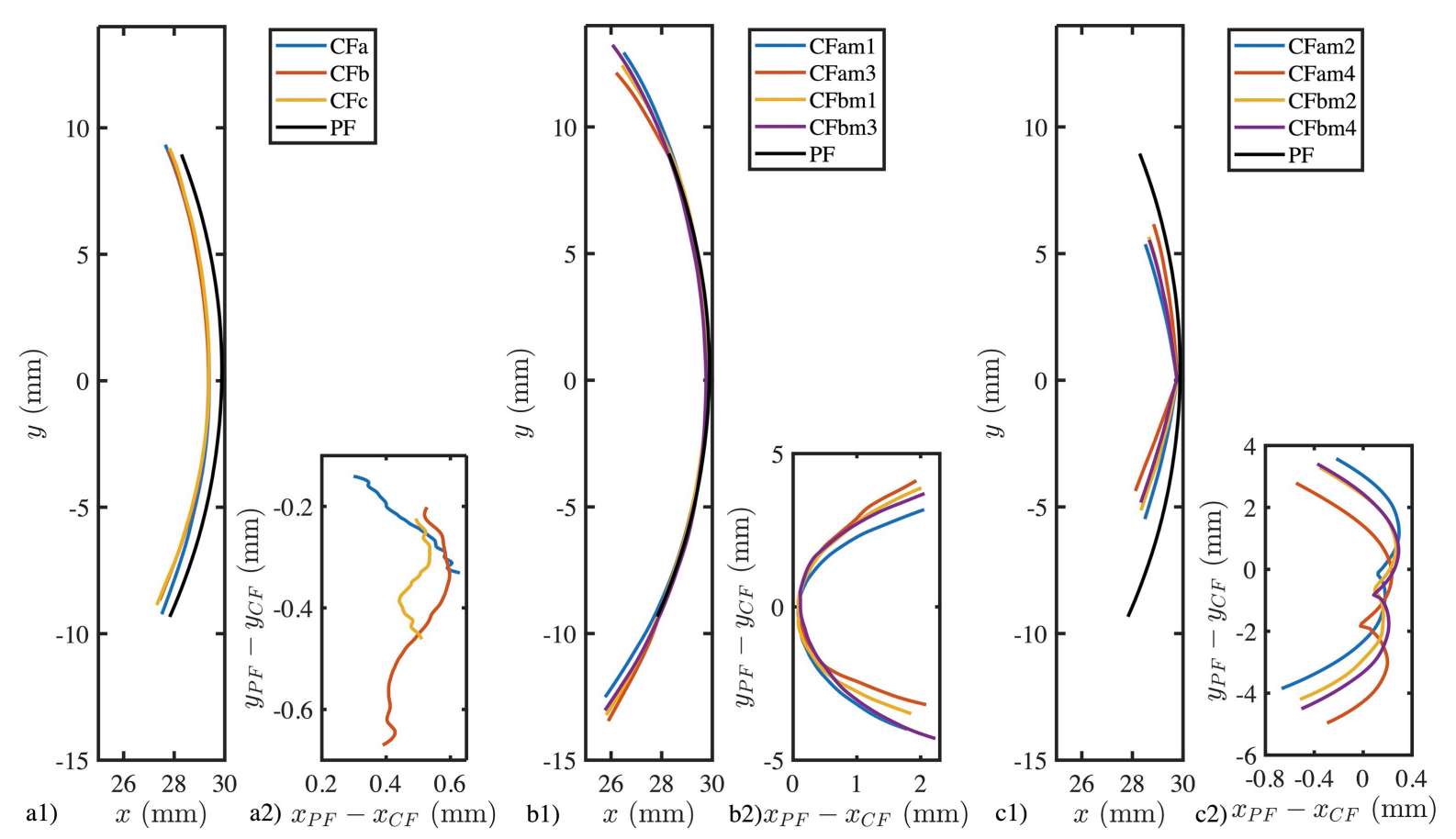

Fig. 8: (1) CF and PF tip positions $(x, y)$ together with (2) Differences between the PF and CF tip positions $\left(x_{P F}-x_{C F}, y_{P F}-y_{C F}\right)$ for (a) Internal, (b) Fixed end and (c) Actuated end cross-over point for actuator extensions $\pm 45 \mathrm{~mm}$

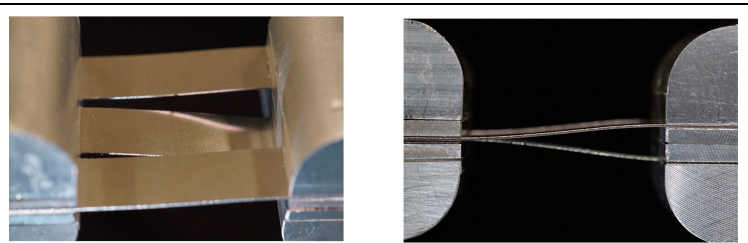

(a) CFam1; triple-section (2 upper) with cross-over point at left fixed end

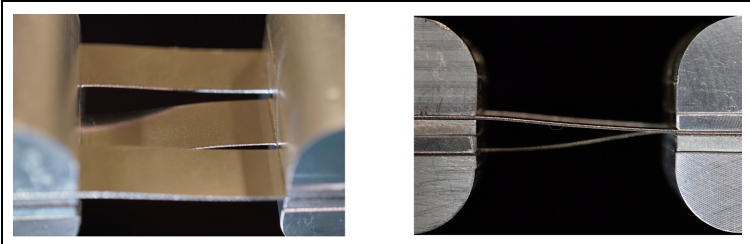

(b) CFam2; triple-section (2 upper) with cross-over point at right actuated end
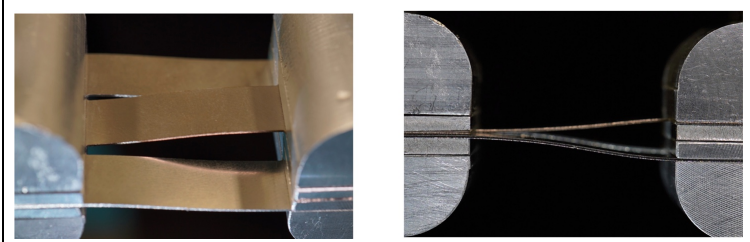

(c) CFam3; triple-section (1 upper) with cross-over point at left fixed end
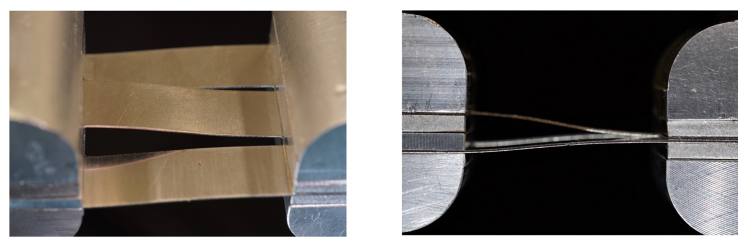

(d) CFam4; triple-section (1 upper) with cross-over point at right actuated end

Fig. 7: Designs of CF having a common end point 


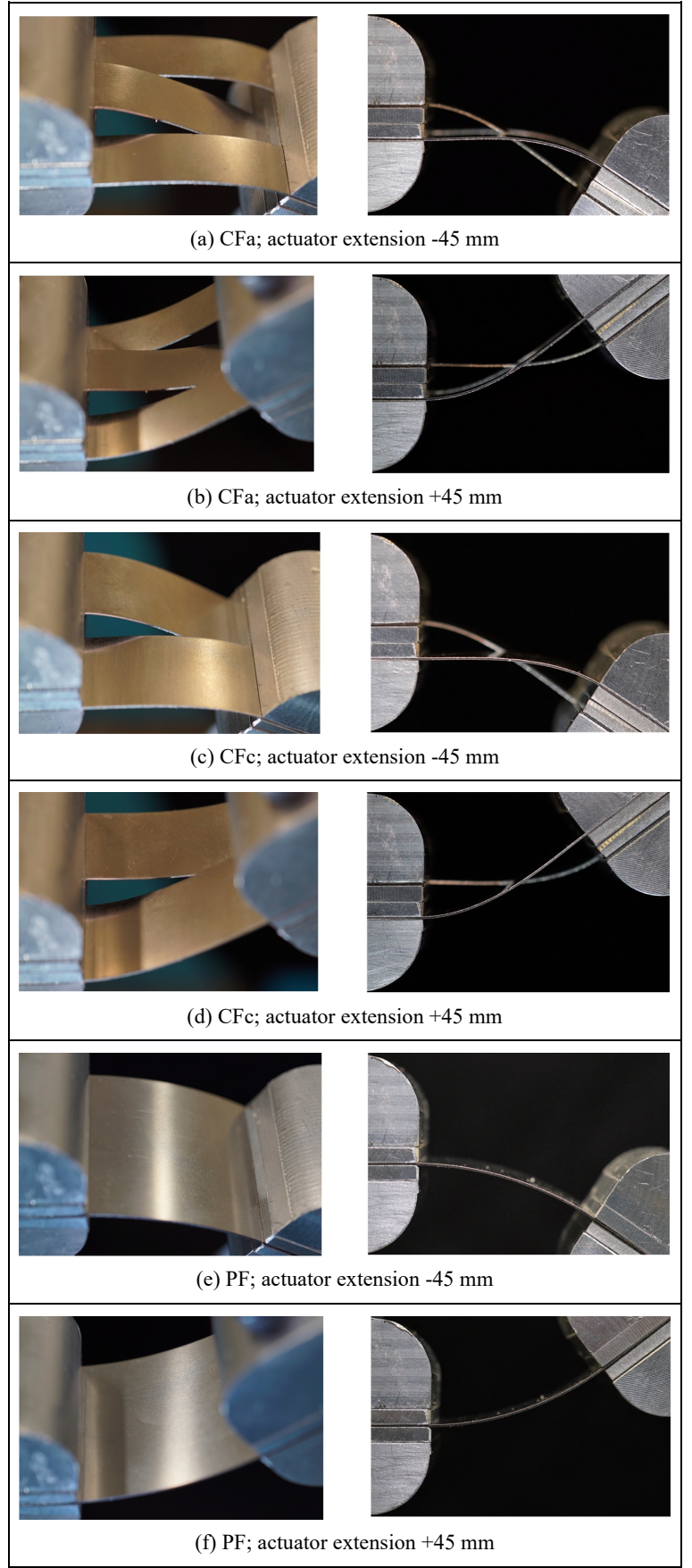

Fig. 9: Deformation of $\mathrm{CFa}, \mathrm{CFc}$ and $\mathrm{PF}$ for minimum and maximum actuator extension $( \pm 45 \mathrm{~mm})$; side and angled views

Two look-up tables are generated, such that for given values of $l_{01}$ and $l_{02}$, the end position coordinates of the two-CF-rigid body system, $\left(x_{e}, y_{e}\right)$, can be identified. The end coordinates can be written as continuous functions of the actuator extensions, $x_{e}=f\left(l_{01}, l_{02}\right)$ and $y_{e}=g\left(l_{01}, l_{02}\right)$.

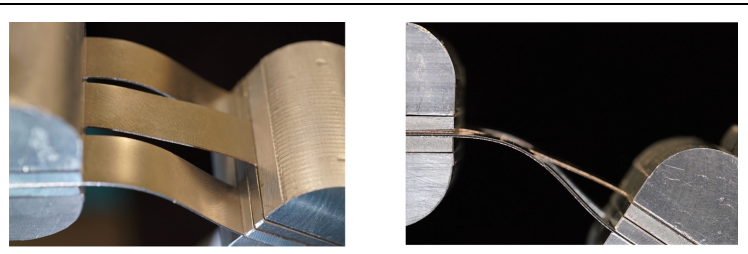

(a) CFam3; actuator extension $-45 \mathrm{~mm}$

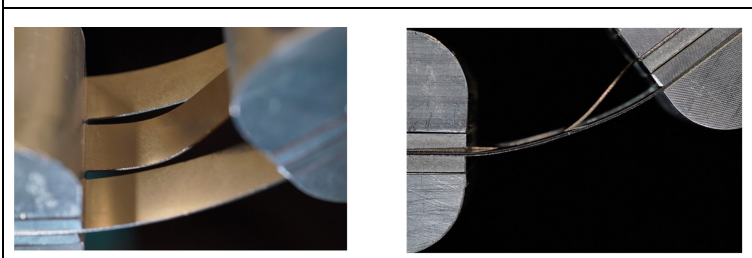

(b) CFam3; actuator extension $+45 \mathrm{~mm}$

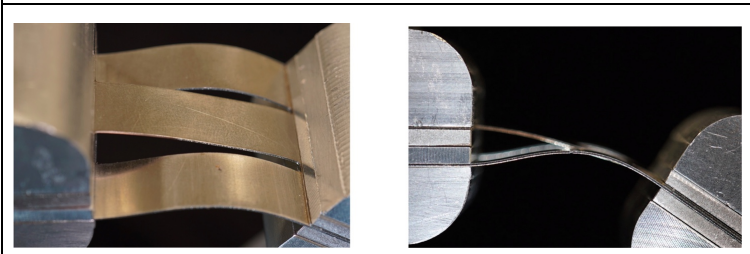

(c) CFam4; actuator extension $-45 \mathrm{~mm}$
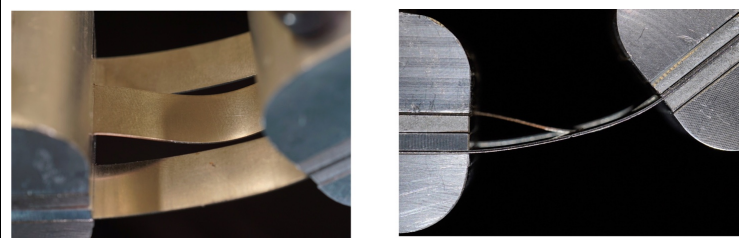

(d) CFam4; actuator extension $+45 \mathrm{~mm}$

Fig. 10: Deformation of CFam 3 and CFam 4 for minimum and maximum actuator extension $( \pm 45 \mathrm{~mm})$; side and angled views

The look-up tables were then exploited to find the actuator extensions needed for a prescribed end position of the system. For example, given a desired end position of $x_{p}=1015 \mathrm{~mm}, y_{p}=0 \mathrm{~mm}$, a contour from each of the lookup tables is found where $x_{e}=x_{p}$ and $y_{e}=y_{p}$. The two resulting contours have been superimposed in Fig. 12 and the points at which the two contour plots intersect are the actuator extensions needed to achieve the desired end position $\left(x_{p}, y_{p}\right)$. There are two solutions, depending on whether the system is positioned above or below the horizontal. This methodology can then be utilised for each position along the trajectory to identify the necessary actuator extension for the end of the two-CF-rigid body system to follow the desired trajectory. The dynamic effects of the system are assumed negligible as the system is moving relatively slowly.

\section{Trajectory Control}

An experimental programme was undertaken to examine the different CFs and their ability to follow a prescribed trajectory. 


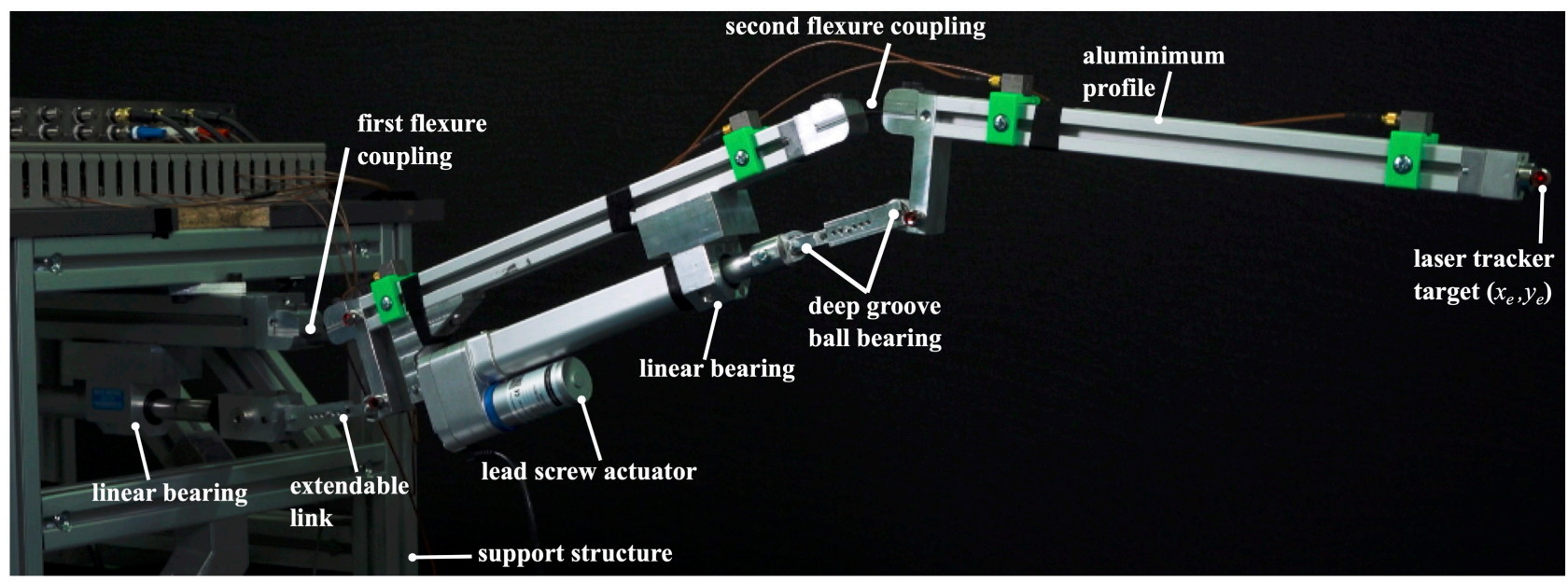

Fig. 11: Experimental facility for two-flexure-coupling rigid body system

Feedforward controllers were developed for each of the triple-section CFs, together with the PF. The extension of the actuator extension was controlled over time using proprietary hardware and controllers designed in the control methodology section. The end position of the system was measured over the complete movement using the 3D laser tracker target positioned at the end of the second rigid body link $\left(x_{e}, y_{e}\right)$ as shown in Fig. 11.

The desired trajectory of the end of the two-CF-rigid body system is that of a straight line between $+200 \mathrm{~mm}$ and $-200 \mathrm{~mm}$ over $14 \mathrm{~s}$ following a sine curve trajectory, $y_{p}=0.2 \sin (2 \pi t+\pi / 2) \mathrm{mm}$, at $x_{p}=1015 \mathrm{~mm}$. The $3 \mathrm{D}$ laser tracker was used to measure the resultant dynamic end position for all cases investigated. The linear actuators used to drive the system exhibited substantially nonideal performance, including drift, backlash and vulnerability to signal noise. Therefore, to ensure that the actuators followed the feedforward control path as closely as possible, they were operated under a proportional feedback loop. The actual actuator extensions were measured by onboard potentiometers and this signal was then subtracted from that of the extension command for the feedforward control scheme. An error signal was then formed and it was this signal on which the feedback loop operated. The gain value used in the feedback loop was a compromise between being sufficiently small to avoid amplifying imperfections in the actuator characteristics and adequately large to enable the system to have favourable tracking performance.

Initially, $\mathrm{CFa}, \mathrm{CFb}$ and the $\mathrm{PF}$ were examined under their respective feedforward control, with the end position of the two-CF-rigid body system given in Fig. 13. The results show that the paths taken by the end of the system for the $\mathrm{CFa}, \mathrm{CFb}$ and $\mathrm{PF}$ are close to that of the desired trajectory, but have some error: $\left[\begin{array}{l}x_{e} \\ y_{e}\end{array}\right]=\boldsymbol{g}\left(\left[\begin{array}{l}x_{p} \\ y_{p}\end{array}\right]\right),\left[\begin{array}{l}\varepsilon_{x} \\ \varepsilon_{y}\end{array}\right]=\left[\begin{array}{l}x_{p}-x_{e} \\ y_{p}-y_{e}\end{array}\right]$,

where $g$ is a flexure coupling-dependent function. The maximum error from the straight line is $\sim 3 \mathrm{~mm}$ in the $x$ direction and $\sim 15 \mathrm{~mm}$ in the $y$ direction.

The resulting trajectories for the $\mathrm{CFa}$ and $\mathrm{CFb}$ modifications under feedforward control derived from their respective empirical data are shown in Fig. 14 and 15. Modifications $\mathrm{m} 1$ and $\mathrm{m} 3$ have similar behaviour for each $\mathrm{CF}$, as do $\mathrm{m} 2$ and $\mathrm{m} 4$. $\mathrm{CFa}$ and $\mathrm{CFb}$ modifications follow similar trends but with small variations for $y<0$. For all $\mathrm{CFa}$ and $\mathrm{CFb}$ modifications, the maximum error is $\sim 4 \mathrm{~mm}$ in the horizontal and $\sim 15 \mathrm{~mm}$ in the vertical, with $\mathrm{CFa}$ giving a larger error than $\mathrm{CFb}$, in general.

The open-loop feedforward control is based on an identified system model, which may possess some uncertainty due to a number of factors. These include imperfect actuator control, the static data utilised in the look-up table being taken without the rigid bodies attached, look-up table interpolation errors and the modular system having minor differences when assembled with the different CFs. Additional path correction may be implemented to achieve more accurate tracking.

The system with a $\mathrm{CFa}, \mathrm{CFb}$ and $\mathrm{PF}$ was investigated to examine if improvements in the feedforward control could lead to better resultant path trajectories. To account for errors in the feedforward control, a new modified feedforward control scheme was derived with path correction:

$\left[\begin{array}{l}\hat{x}_{e} \\ \hat{y}_{e}\end{array}\right]=g\left(\left[\begin{array}{c}x_{p}+\varepsilon_{x} \\ y_{p}+\varepsilon_{y}\end{array}\right]\right)=g\left(\left[\begin{array}{c}2 x_{p}-x_{e} \\ 2 y_{p}-y_{e}\end{array}\right]\right)$. 


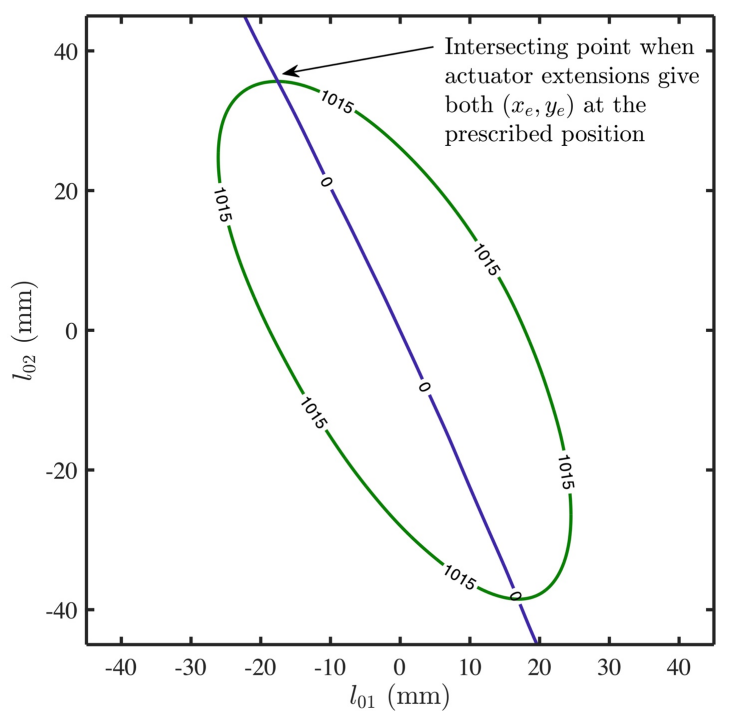

Fig. 12: Contour for values of the first and second actuator extensions, $l_{01}$ and $l_{02}$, respectively, for $x_{e}=x_{p}=1015 \mathrm{~mm}$ and $y_{e}=y_{p}=0 \mathrm{~mm}$
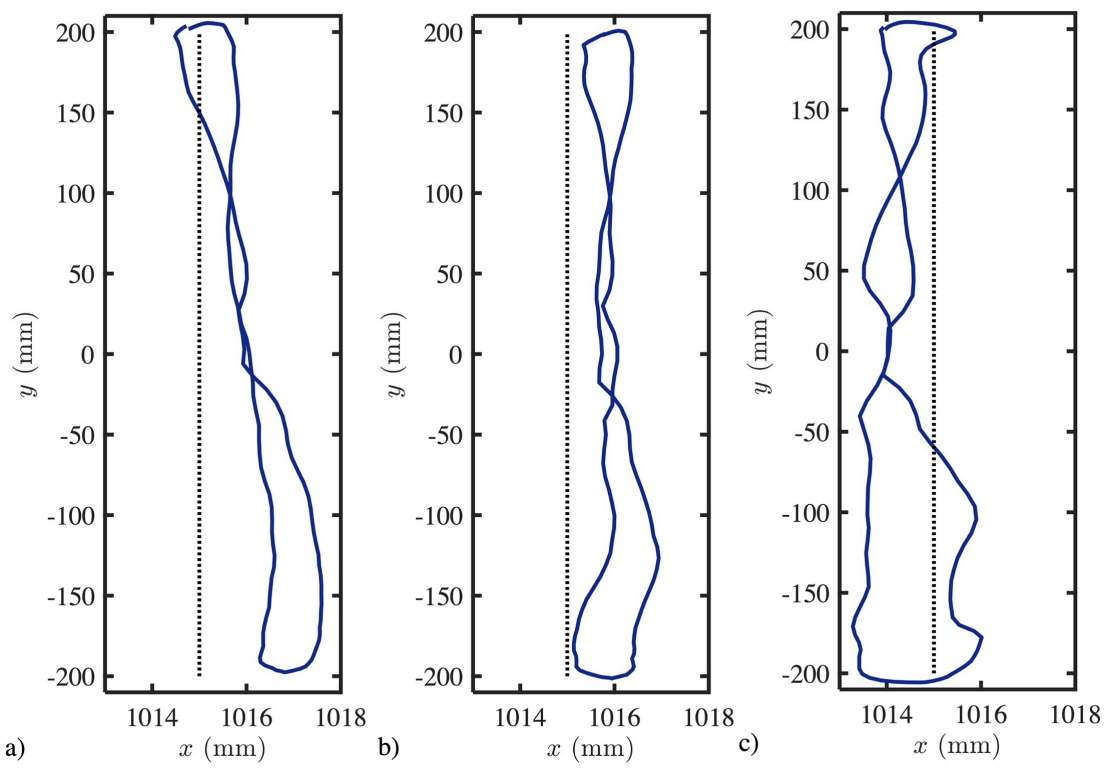

Fig. 13: Dynamic trajectories at the end of the two-CF-rigid body system for (a) $\mathrm{CFa}$, (b) $\mathrm{CFb}$ and (c) $\mathrm{PF}$

This new demand trajectory $\left(x_{p}+\varepsilon_{x}, y_{p}+\varepsilon_{y}\right)$ was then used with the same control methodology to identify adjusted displacement profiles for the actuator rods. Figure 16 shows the original and adjusted trajectories of the $\mathrm{CFa}, \mathrm{CFb}$ and $\mathrm{PF}$ cases, with Fig. 17 showing the errors in the vertical and horizontal planes against time. The corresponding maximum errors for each case in the horizontal and vertical planes are given in Table 1, for both the original and adjusted trajectories. The results show a significant improvement in performance with the modified feedforward control scheme with path correction (adjusted) where the maximum error from the desired trajectory being reduced by at least $73 \%$ and $71 \%$ in the $(x, y)$ directions, for a CF and by $62 \%$ and $86 \%$ in the $(x, y)$ directions, for a PF. The remaining errors between the demand trajectory and the realised paths are residual from the non-smooth actuation/actuator capabilities. 

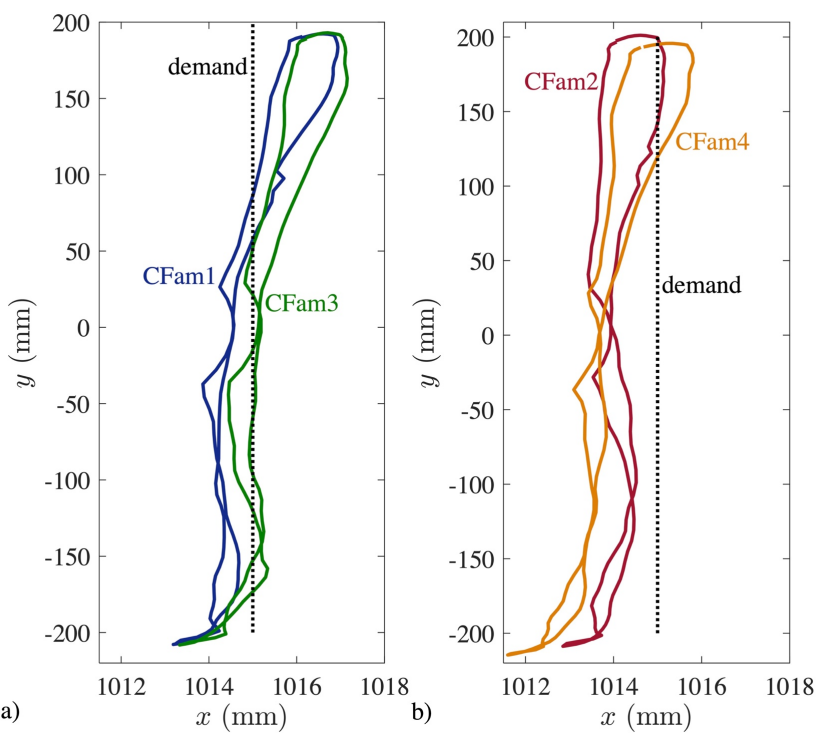

Fig. 14: Dynamic trajectories at the end of the two-CF-rigid body system for (a) CFam1 and CFam 3 and (b) CFam2 and CFam4
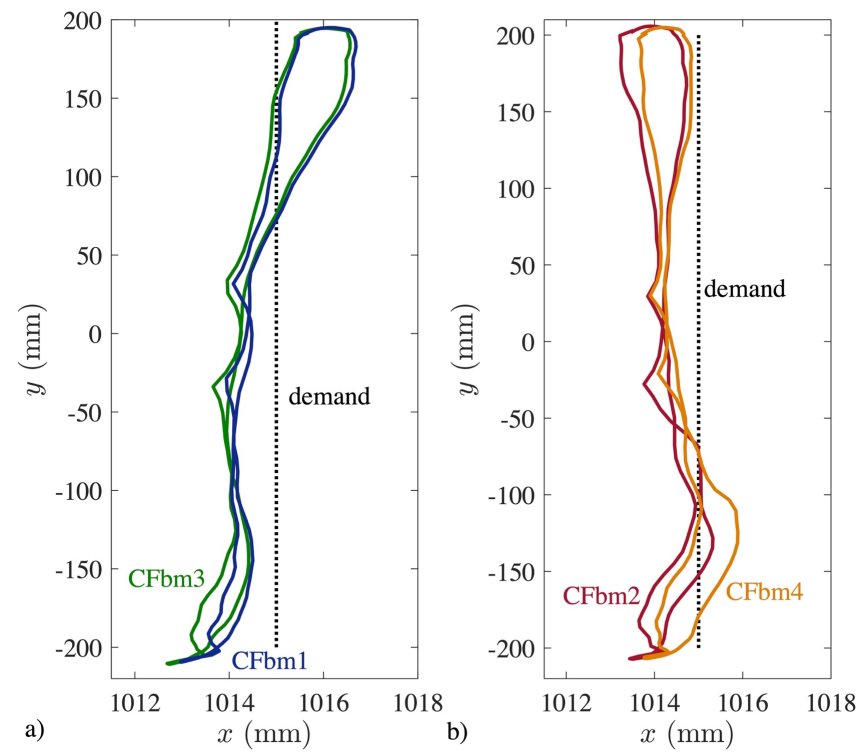

Fig. 15: Dynamic trajectories at the end of the two-CF-rigid body system for (a) CFbm1 and CFbm3 and (b) CFbm2 and CFbm4

Table 1: Maximum errors from the desired trajectory for $\mathrm{CFa}, \mathrm{CFb}$ and $\mathrm{PF}$ in horizontal $(x)$ and vertical planes $(y)$ for the original and adjusted trajectories

\begin{tabular}{|c|c|c|c|c|c|c|}
\hline \multirow[b]{2}{*}{ Design } & \multicolumn{2}{|l|}{ Original } & \multicolumn{2}{|c|}{ Adjusted } & \multicolumn{2}{|c|}{$\%$ Reduction } \\
\hline & $x(\mathrm{~mm})$ & $\mathrm{a} y(\mathrm{~mm})$ & $x(\mathrm{~mm})$ & $y(\mathrm{~mm})$ & $x$ & $y$ \\
\hline $\mathrm{CFa}$ & 2.59 & 14.8 & 0.705 & 4.29 & 73 & 71 \\
\hline $\mathrm{CFb}$ & 1.94 & 11.9 & 0.445 & 2.32 & 77 & 80 \\
\hline PF & 1.72 & 22.9 & 0.647 & 3.20 & 62 & 86 \\
\hline
\end{tabular}



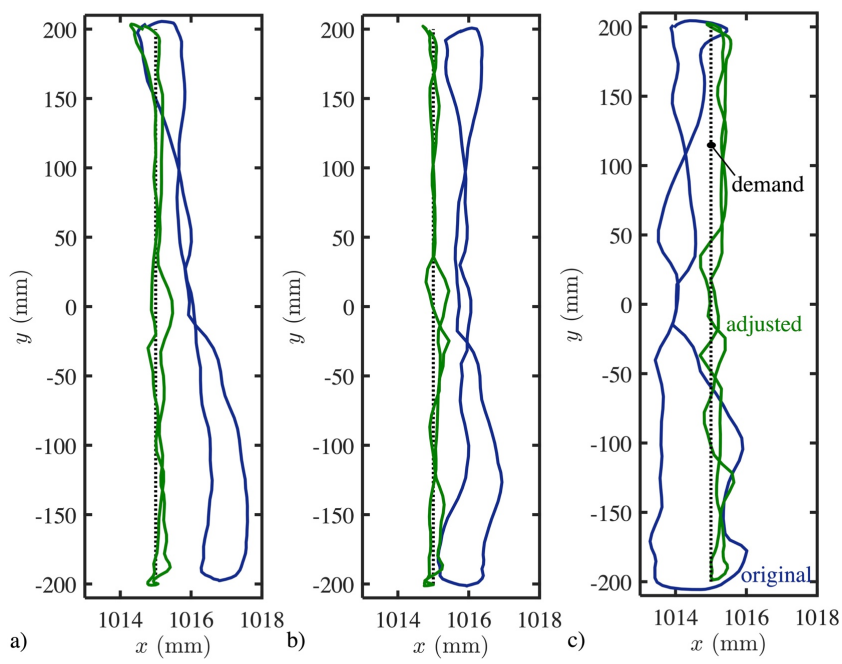

Fig. 16: Dynamic trajectories at the end of the two-CF-rigid body system under adjusted control for (a) $\mathrm{CFa}$, (b) $\mathrm{CFb}$ and (c) $\mathrm{PF}$
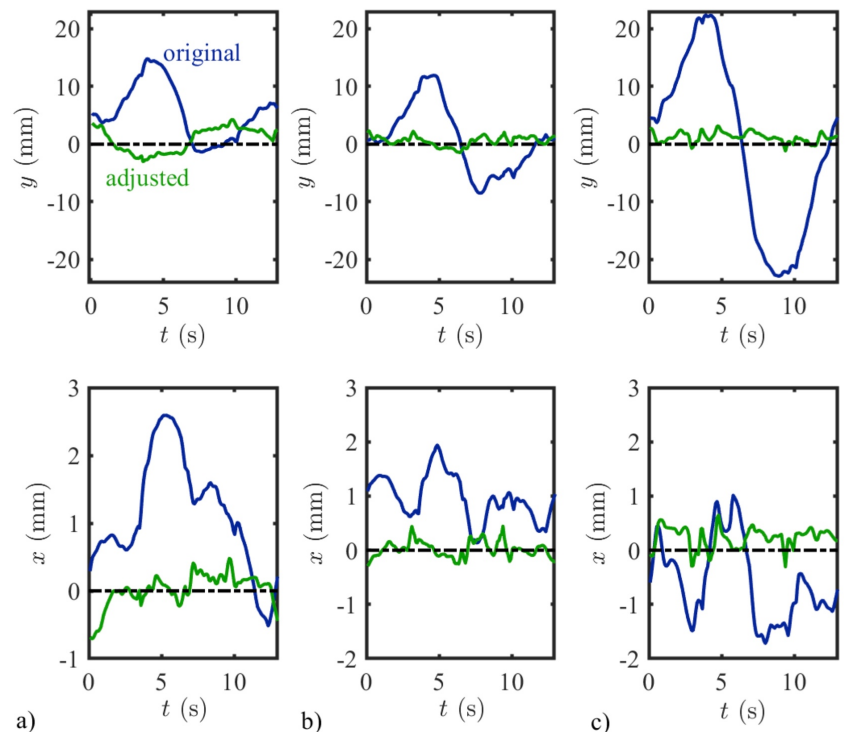

Fig. 17: Errors in the dynamic trajectories at the end of the two-CF-rigid body system in the vertical and horizontal planes against time under adjusted control for (a) $\mathrm{CFa}$, (b) $\mathrm{CFb}$ and (c) $\mathrm{PF}$

Table 2: Key features of the different designs and modifications considered

\begin{tabular}{lll}
\hline Design & Advantages & Disadvantages \\
\hline $\mathrm{PF}$ & Simple design/construction & $\begin{array}{l}\text { Least robust } \\
\text { Extra degrees of freedom }\end{array}$ \\
\hline $\mathrm{CFa}$ & $\begin{array}{l}\text { More resistant to torsion } \\
\mathrm{CFb}\end{array}$ & $\begin{array}{l}\text { More complex design } \\
\text { End clamping more intricate }\end{array}$ \\
& Section width insignificant & \\
\hline $\mathrm{CFc}$ & Least error in trajectory & Torsion/out of plane motion \\
& - & Not feasible \\
\hline $\mathrm{m} 1 / \mathrm{m} 3$ & Largest range of motion & - \\
\hline $\mathrm{m} 2 / \mathrm{m} 4$ & - & Smallest range of motion \\
& & Sharp transition through $\theta=0$ \\
\hline
\end{tabular}




\section{Discussion}

From the current work, the following outcomes should be highlighted, which are summarised in Table 2. The CFc flexure should not be used because it experiences torsional effects, thus out of plane motion, due to the design imposing a moment on the flexure clamp at the actuated end. From examining the $\mathrm{CFa}$ and $\mathrm{CFb}$ flexure, along with their modifications, it can be concluded that the widths of individual sections do not have a significant difference on the CF behaviour, provided the overall width remains the same. Analysing the effect of the cross-over point position demonstrates that when the cross-over point is at the actuated end (modifications $\mathrm{m} 2$ and $\mathrm{m} 4$ ), the flexure coupling has sharp and distinct changes at the horizontal point, together with a reduced range of motion, resulting in it being nonideal for use. A CF with the cross-over point at the fixed end (modifications $\mathrm{m} 1$ and $\mathrm{m} 3$ ), has a larger range of motion than when the cross-over point is at the centre, but either would be appropriate to use, along with a PF. The advantages of the $\mathrm{CF}$ are that they are more resistant to torsional effects, the pivot moves less along the length of the flexure, giving a better representation of a traditional hinge and has multiple sections in case of one section failing. However, they have a more complex design/construction than a PF, but a PF has extra degrees of freedom due to the larger width allowing for greater potential torsional effects.

\section{Conclusion}

Compound Flexure couplings (CFs), comprising multiple sections of spring steel, have been analysed together with a Plain Flexure coupling (PF). During the theoretical study of a PF based on large deformation Euler-Bernoulli theory, it was identified that there is a many-to-many relationship between the pair of forces and moment applied to the tip of the flexure and the resulting tip deflection. Therefore, modelling of a $\mathrm{CF}$ is not straightforward and as such different designs of the CFs were characterised using a bespoke experimental facility. Appropriate designs of CFs can be used to prevent torsion and out of plane motion. Furthermore, the range of deflection of the CFs for given actuator extensions is significantly affected by the crossover point of the flexure sections. However, the widths of the sections have a negligible effect on the CF behaviour.

The behaviour of a PF was examined with the deflection identified by experimental measurements and predicted by the theoretical models shown to have good agreement. Therefore, the feedforward control methodology is based on identified models using experimental data; this control approach negates the need for complex and expensive models to be formulated for CFs, while still providing control of equal accuracy. The ability to control a two-CF-rigid body system was investigated through feedforward control based on the static test data and proportional feedback of actuator rod extensions. Controllers were formulated for each $\mathrm{CF}$ design and implemented in the system for the end point to follow a vertical straight line. Applying the corresponding feedforward control to the system containing the CFs or PF gave the realised trajectories and tracking errors. These trajectories were repeatable over multiple cycles. Reduction of the realised tracking errors with the CFs and PF was achieved using adjusted input profiles for the actuator extensions, derived from the initial realised trajectories, with reductions in tracking errors by at least $73 \%$ and $71 \%$ in the $(x, y)$ directions, respectively, for the CFs considered and by $62 \%$ and $86 \%$ in $(x, y)$ directions, respectively, for a PF. Therefore, it has been demonstrated that systems containing CFs can be controlled through feedforward control schemes, without any necessity of modelling of the complex CFs.

\section{Acknowledgement}

Photography credit to Simon Wharf of the AV unit at the University of Bath.

\section{Funding Information}

The authors are grateful for funding from the EPSRC, under grant no. EP/M016285/1.

\section{Author's Contributions}

All authors designed the research programme.

Nicola Y. Bailey: Developed the numerical simulations. Nicola Y. Bailey, Christopher Lusty and Patrick S. Keogh: Performed the experimental test under guidance of Patrick S. Keogh. All authors contributed to the preparation of the manuscript and approval for submission.

\section{References}

Argyris, J. H., \& Symeonidis, S. (1981). Nonlinear finite element analysis of elastic systems under nonconservative loading-natural formulation. Part I. Quasistatic problems. Computer Methods in Applied Mechanics and Engineering, 26(1), 75-123. doi.org/10.1016/0045-7825(81)90131-6

Bailey, N. Y., Lusty, C., \& Keogh, P. S. (2018). Nonlinear flexure coupling elements for precision control of multibody systems. Proceedings of the Royal Society A: Mathematical, Physical and Engineering Sciences, 474(2218), 20180395. doi.org/10.1098/rspa.2018.0395

Chen, G., Liu, P., \& Ding, H. (2020). Nonlinear static modeling of a tip-tilt-piston micropositioning stage comprising leaf-spring flexure hinges. Proceedings of the Institution of Mechanical Engineers, Part C: Journal of Mechanical Engineering Science, 234(10), 1969-1978. doi.org/10.1177/0954406220902173 
Fantner, G. E. (2006). Components for high speed atomic force microscopy. Review of Scientific Instruments, 106: 881-887. doi.org/10.1016/j.ultramic.2006.01.015

Guo, J., \& Lee, K. M. (2013). Compliant joint design and flexure finger dynamic analysis using an equivalent pin model. Mechanism and Machine Theory, 70, 338-353. doi.org/10.1016/j.mechmachtheory.2013.08.001

Henning, S., \& Zentner, L. (2021). Analysis of planar compliant mechanisms based on non-linear analytical modeling including shear and lateral contraction. Mechanism and Machine Theory, 164, 104397. doi.org/10.1016/j.mechmachtheory.2021.104397

Henning, S., Linß, S., Gräser, P., Theska, R., \& Zentner, L. (2021). Non-linear analytical modeling of planar compliant mechanisms. Mechanism and Machine Theory, 155, 104067. doi.org/10.1016/S0094-114X(01)00007-6

Herfst, R., Dekker, B., Witvoet, G., Crowcombe, W., de Lange, D., \& Sadeghian, H. (2015). A miniaturized, high frequency mechanical scanner for high speed atomic force microscope using suspension on dynamically determined points. Review of Scientific Instruments, 86(11), 113703. doi.org/10.1063/1.4935584

Howell, L. L. (2001). Compliant Mechanisms. Wiley, New York. doi.org/10.1063/1.4935584

Howell, L. L., \& Midha, A. (1994). A method for the design of compliant mechanisms with small-length flexural pivots. doi.org/10.1115/1.2919359

Kimiaeifar, A., Lund, E., Thomsen, O. T., \& Sørensen, J. D. (2011). Application of the homotopy analysis method to determine the analytical limit state functions and reliability index for large deflection of a cantilever beam subjected to static co-planar loading. Computers \& Mathematics with Applications, 62(12), 4646-4655.

doi.org/10.1002/num.20538

Liarokapis, M. V., \& Dollar, A. M. (2016). Post-contact, in-hand object motion compensation with adaptive hands. IEEE Transactions on Automation Science and Engineering, 15(2), 456-467.

doi.org/10.1109/ROMAN.2016.7745229

Liu, M., Zhang, X., \& Fatikow, S. (2017). Design of flexure hinges based on stress-constrained topology optimization. Proceedings of the Institution of Mechanical Engineers, Part C: Journal of Mechanical Engineering Science, 231(24), 4635-4645. doi.org/10.1177/0954406216671346

Lobontiu, N. (2001). Distributed-parameter dynamic model and optimized design of a four-link pendulum with flexure hinges. Mechanism and Machine Theory, 36, 653-669.

doi.org/10.1016/S0094-114X(01)00007-6.
Ma, F., \& Chen, G. (2016). Modeling large planar deflections of flexible beams in compliant mechanisms using chained beam-constraint-model. Journal of Mechanisms and Robotics, 8(2), 021018. doi.org/10.1115/1.4031028

Ma, W., Wang, R., Zhou, X., \& Meng, G. (2020). The performance comparison of typical notched flexure hinges. Proceedings of the Institution of Mechanical Engineers, Part C: Journal of Mechanical Engineering Science, 234(9), 1859-1867.

doi.org/10.1177/0954406219897941

Odhner, L. U., \& Dollar, A. M. (2012). The smooth curvature model: An efficient representation of Euler-Bernoulli flexures as robot joints. IEEE Transactions on Robotics, 28(4), 761-772. doi.org/10.1109/TRO.2012.2193232

Odhner, L. U., Jentoft, L. P., Claffee, M. R., Corson, N., Tenzer, Y., Ma, R. R., ... \& Dollar, A. M. (2014). A compliant, underactuated hand for robust manipulation. The International Journal of Robotics Research, 33(5), 736-752. doi.org/10.1177/0278364913514466

Rao, B. N., Shastry, B. P., \& Rao, G. V. (1986). Large deflections of a cantilever beam subjected to a tip concentrated rotational load. The Aeronautical Journal, 90(897), 262-266. doi.org/10.1017/S0001924000015840

Shvartsman, B. S. (2013). Analysis of large deflections of a curved cantilever subjected to a tipconcentrated follower force. International Journal of Non-Linear Mechanics, 50, 75-80. doi.org/10.1016/j.ijnonlinmec.2012.10.015

Smith, S. T. (2000). Flexures: Elements of elastic mechanisms. Crc Press. doi.org/10.1201/9781482282962

Spiers, A. J., Liarokapis, M. V., Calli, B., \& Dollar, A. M. (2016). Single-grasp object classification and feature extraction with simple robot hands and tactile sensors. IEEE transactions on haptics, 9(2), 207-220. doi.org/10.1109/TOH.2016.2521378

Venkiteswaran, V. K., \& Support, H. J. (2016). A threespring pseudorigid-body model for soft joints with significant elongation effects. Journal of Mechanisms and Robotics, 8(6), 061001. doi.org/10.1115/1.4032862

Wang, J., Chen, J. K., \& Liao, S. (2008). An explicit solution of the large deformation of a cantilever beam under point load at the free tip. Journal of computational and applied mathematics, 212(2), 320-330. doi.org/10.1016/j.cam.2006.12.009 